\title{
LE PRINCIPE DE CIRCULATION ET L'ÉCHEC DE LA MYTHOLOGIE TRANSEUROPÉENNE
}

\author{
Henriette AssÉo
}

RÉSUMÉ : Nous avons cherché à identifier un aspect du refoulement du cosmopolitisme des Lumières, complémentaire de la fabrique des identités nationales en Europe au XIX ${ }^{\mathrm{e}}$ siècle et dans l'entre-deux-guerres. L'idée de civilisation commune serait maintenue par la capacité réciproque de traduction des langues nationales, du fait de leurs origines communes indo-européennes. L'utopie de la langue remplacerait le parcours matériel des Lumières. C'est l'échec de la construction du mythe indianiste européen et non pas son succès qui explique le dévoiement idéologique des relations entre langue et nation. La promotion manquée de la langue tsigane dans le monde académique illustre bien cet échec. Après la Première Guerre mondiale, la transformation du principe des nationalités en ethnopolitique fit disparaître jusqu'au souvenir de cette ambition commune.

Mots-CLÉs : histoire de l'Europe, Tsiganes, indo-européanisme.

ABSTRACT : We have sought to identify an aspect of the repression of Enlightenment cosmopolitanism complementary to the creation of national identities in XIX ${ }^{\text {th }}$-century Europe and the intervening war period. The idea of a common civilization would be maintained by the reciprocal capacity of translating national languages, because of their Indo-European common origins. The utopia of the language would replace the material route of the Enlightenment. It is the failure of the construction of European Indianist myth and not its success which explains the ideological diverting of the relations between language and nation. The missed promotion of the Gypsy language in the academic world illustrates this failure perfectly. After the First World War, the transformation of the principle of nationalities in ethnopolitics removed even the memory of this common ambition.

KEYWORDS : history of Europe, Gypsies, Indo-Europeanism. 
ZuSAMmENFASSUNG : Wir haben versucht, einen Aspekt der Verdrängung des Kosmopolitismus der Aufklärung zu identifizieren. Dieser Aspekt ist als Begleiterscheinung zur Entstehung der nationalen Identitäten in Europa im neunzehnten Jahrhundert und in der Zwischenkriegszeit zu verstehen. Die Idee einer gemeinsamen Kultur hätte sich durch die Möglichkeit einer gegenseitigen Übersetzung der nationalen Sprachen aufgrund ihres gemeinsamen indogermanischen Ursprungs bewähren sollen. Die Sprachutopie hätte die materielle Anschauung der Aufklärung ersetzen sollen. Die Ideologisierung des Verhältnisses zwischen Sprache und Nation erklärt sich durch das Scheitern der Errichtung des europäischen indogermanischen Mythos und nicht durch dessen Erfolg. Die verfehlte Beförderung von der Sprache der Zigeuner auf akademisches Niveau illustriert deutlich dieses Scheitern. Nach dem ersten Weltkrieg liess die Verwandlung dieses Prinzips der Nationalitäten in eine Ethnopolitik sogar die Spuren dieses gemeinsamen Projektes verschwinden.

STICHWÖRTER : europäische Geschichte, Zigeuner, Indogermanistik.

Henriette Asséo, née en 1947, est professeur agrégée à l'École des hautes études en sciences sociales à Paris. Ses travaux portent sur l'histoire des Tsiganes et sur l'imaginaire de la mobilité en Europe. Dans le cadre du Centre de recherches tsiganes de l'université Paris VRené Descartes, avec le soutien des Communautés européennes et du Conseil de l'Europe, elle a dirigé la collection Interface, formée d'ouvrages originaux sur l'histoire des Tsiganes, publiés simultanément dans plusieurs pays d'Europe.

Adresse : École des hautes études en sciences sociales, Centre de recherches historiques, 54 boulevard Raspail, F-75270 Paris Cedex 06.

Courrier électronique : asseo@ehess.fr 
Zgubilo sie pokolenie. Takze miasta. Narody.

«Elle se perdit, ma génération. De même les villes. Les nations. »

Czeslaw Milosz ${ }^{1}$

L'Europe a annoncé sa prophétie autoréalisatrice en se privant délibérément de la référence à la «maison commune » comme principe de civilisation. Pourtant, les colloques sur les identités européennes se sont multipliés depuis la fin des années 1990, et les historiens tentaient d'éclairer le présent à la lumière du passé. Confrontés aux nouveaux enjeux d'une remise en circulation des sociétés européennes depuis la chute du mur de Berlin, ils voudraient assurer leur fonction de gardiens du temple de l'événementiel sans pour autant se livrer à une analyse spectacle de l'Occident $^{2}$. Quand ils s'interrogent sur leur pratique professionnelle des Usages politiques du passé, il peut advenir à certains de verser sur le mode de la déploration dans la prédiction rétrospective, puisque changer de siècle n'est jamais innocent ${ }^{3}$.

Comment parler d'une Europe dont nous voudrions éclairer un paradoxe historique à notre avis déterminant ? Il existe, en effet, à partir du XIX siècle, une opposition entre la circulation comme « grammaire cachée » de la civilisation européenne et le refoulement du cosmopolitisme des Lumières. La tension entre la prétention à la mobilité et sa dénégation forme l'histoire du « principe de circulation » en Europe et de ses métamorphoses. Si cette opération de mise à jour de configurations historiques masquées par les points de vue de la nation n'est pas effectuée, les débats actuels sur la comparaison et le multiculturalisme ne serviraient que des desseins tactiques.

1. Milosz, 1987, p. 9. Ce poème ouvre le recueil des six conférences sur la littérature, prononcées par l'auteur à l'université de Harvard pendant l'année universitaire 1981-1982.

2. Le projet de la morphologie historique, appelée aussi morphologie culturelle offre la possibilité de disposer les civilisations selon des formes décryptables, mais le recours à l'esthétique comme principe interprétatif ne satisfait pas l'historien. Voir MANCINI, 1999.

3. BASCHET, 2001. Nous souscrivons pleinement à la phrase de conclusion, ibid., p. 74 : «L'histoire n'expliquera jamais le présent et n'éclaire plus le futur. Mais, plus que jamais, elle a pour mission de restaurer d'un même mouvement un espace d'expérience, nécessaire à la saisie du contemporain, et un horizon d'attente, dont la promesse est certaine mais la nature indéterminée. » 
Pourtant, la comparaison historique exploite de façon implicite des jeux de proximité de civilisation. Ce qui peut paraître normal au médiéviste, au moderniste ou à l'historien de l'Antiquité devient, à mesure que l'on se rapproche de notre univers contemporain, très problématique ${ }^{4}$. Or, l'émergence de nouveaux objets historiques ne peut advenir de la comparaison entre des objets préconstruits. Les historiens repèrent en les rapprochant et en les mettant à distance des conjonctures agencées en objets complexes à condition de baliser les attendus souterrains des représentations mentales collectives.

Existe-t-il dans ces conditions une possibilité de production historique qui « dénationalise » les objets en histoire contemporaine sans « dénationaliser » les attitudes intellectuelles ? Les historiens du contemporain ont marqué jusqu'à présent une nette défiance à l'égard de ce type d'entreprise. Les discours et les représentations politiques sont analysés à partir des découpages nationaux. Encombrant Léviathan, l'État national est posé comme le cadre territorial naturel d'exercice des sociétés européennes et de leur culture et, par là même, tend à forcer le trait de discontinuités surdéterminées par le primat du politique.

La méfiance que montrent les historiens du contemporain à parler de civilisation européenne puise aussi à l'ordre du vécu. La description minutieuse des vicissitudes de «l'équilibre européen » par les professeurs de la vieille Sorbonne n'éveillait rien d'exaltant dans l'imagination de la génération d'aprèsguerre. Mais la précédente considérait, pour d'autres raisons, avec méfiance les envolées sur la civilisation européenne. Elle avait appris à ses dépens que la pensée traditionaliste, contemptrice des « idées françaises », dont l'expansion aurait conduit à sa ruine un continent abandonné par la Providence, avait constamment sous la plume le vocable de civilisation européenne comme substitut du terme de civilisation chrétienne.

\section{LA « GRAMMAIRE CACHÉE » DE LA CIRCULATION EUROPÉENNE}

S'il existe de multiples usages de la référence mentale à l'Europe, les textes et les pratiques montrent un surprenant mélange entre l'indécision dans la définition pratique et un universalisme moral. Cette manifestation d'une identité collective supérieure aux fabriques nationales se présente pourtant comme un motif rhétorique récurrent. La civilisation européenne serait le lieu de la présence immanente d'une Providence sécularisée, distribuant entre les nations, à l'aune de la nouvelle technicité, les bons points, entre rédemption historique et décadence.

4. Brague, 1999. 
Tous les idéologues patentés de la nation, indépendamment de leurs options politiques, se livrent à une déclinaison de ce motif. Ainsi, Ernest Renan prend pour pivot de sa réflexion sur le devenir des sociétés, non pas la nation, mais une allusion ambiguë à l'Europe comme critère de la supériorité interne de la civilité occidentale. Comme le fait remarquer Raoul Girardet, le témoignage exposé dans La Réforme intellectuelle et morale sur la crise de la conscience française au lendemain de la défaite de 1870 parle aussi de l'avènement d'un nouvel ordre européen ${ }^{5}$. Renan pensait que la politique bismarckienne, en entraînant l'irrémédiable hostilité entre la France et l'Allemagne, mettait en cause les fondements spirituels caractéristiques de la civilisation européenne, à partir desquels se définit le concept même de nation, et il affirmait : «L'Europe est une confédération d'États réunis par l'idée commune de la civilisation ${ }^{6}$.» On verra, selon lui, la fin de la guerre quand, au principe des nationalités, on joindra le principe qui en est le correctif, celui de la fédération européenne.

Comment concilier, de part et d'autre du Rhin, l'effort de pédagogie du National et la pensée d'une Europe de la circulation ? Les échanges savants entre la France et l'Allemagne n'ont pas été interrompus par les confrontations militaires de 1870. Le véritable tournant se situerait plutôt vers 1907, et la montée des mystiques nationales s'expliquerait par l'abandon de la référence à l'Europe comme «communauté imaginée », au sens de Benedict Anderson. Comparant l'Allemagne de 1890 et celle de 1907, Fernand Baldensperger évoquait en 1935 la crainte que suscitaient déjà à cette époque les constellations nouvelles de l'Allemagne :

« Herriot croyait qu'on intimide l'Allemagne avec des politesses : en fit-il, ce même automne 1907, au congrès d'hygiène de Hambourg où vint figurer le maire de Lyon ! Et, en général, d'anciens dreyfusards devenus les chefs de l'historiographie française, G. Monod, Aulard, Seignobos, se refusaient à étudier sur le terrain, pourrait-on dire, c'est-à-dire au voisinage des empires, l'histoire des temps modernes, et laissaient ce soin à Jacques Bainville, dès lors contraint de faire la jonction avec l'Action française ${ }^{7}$. »

La critique est trop sévère puisque Charles Seignobos participait, avec Miguel de Unamuno, en 1902, au Comité de l'union des nationalités, ce qui montrait l'attention des historiens français à la politique des nationalités.

Le terme même de civilisation européenne aura servi à définir en creux une manière d'horizon universaliste de territorialité non intériorisée, confortant le

5. Renan, 1996, p. 7-8. Voir Bell, 2002.

6. Renan, 1996, Nouvelle lettre à M. Strauss, Paris, 15 sept. 1871, p. 205-219, en part. p. 212.

7. BALDENSPERGER, 1940, p. 204. Ce texte a été écrit en 1935 avant son départ pour les États-Unis. L'auteur de Goethe en France place en exergue de son chapitre intitulé «La défense d'une civilisation » cette citation de Nietzsche : «Peuple allemand, peuple alarmant... » 
sentiment d'appartenance nationale comme une proximité de mœurs. Mais la réussite nationale impliquait aussi une capacité de traductions des langues européennes les unes dans les autres. L'enjeu linguistique et l'utopie de la langue commune devenaient essentiels. Les réformes scolaires engagées dans tous les États d'Europe, en gros après 1880, ont cantonné cette «passion européenne » au fonds commun des humanités classiques. En reléguant le terreau sensible aux années d'apprentissage, elles laissèrent la place, à l'âge adulte, à l'épanouissement des idéologies nationalistes et régionalistes. Lorsque Nietzsche se livre à une réflexion essentielle sur la langue maternelle, sa critique de la culture allemande ne s'accomplit pas au nom d'un défaut de sacralisation, mais plutôt comme une critique de cette sacralisation : "Ni la langue maternelle, ni les "classiques" ne constituent plus une valeur à laquelle il est nécessaire de se référer. Leur sacralisation devient suspecte, comme tout ce qui s'apparente à la construction imaginaire d'une communauté nationale-spirituelle ${ }^{8}$. »

\section{L'INDIANISME COMME MYTHE TRANSEUROPÉEN ET SON ÉCHEC}

L'un des éléments, à notre avis fondamental, de la "grammaire cachée » européenne serait donc situé dans cette opération de transmutation de l'idéal cosmopolite en une mythologie de l'indo-européanisme. Le parcours éclairé assurant la paix du monde se transformait en un primat utopique de la langue originelle commune assurant dans un même mouvement l'identité nationale et la communication intra-européenne par la traduction. Ce mythe était construit sur l'opposition irréductible entre aryens et sémites, mais le monde sémitique n'était pas défini clairement et sa géographie variait d'un auteur à l'autre ${ }^{9}$. Les penseurs du mythe indo-européen ont voulu donner à la philologie et à la linguistique la puissance mobilisatrice de la philosophie des Lumières. Toutefois, l'impasse théorique entre l'affirmation d'une communauté d'origine par la langue et la différenciation anthropologique en acte dans les constructions nationales du XIX ${ }^{\mathrm{e}}$ siècle constituait un casse-tête qui, à notre avis, a conduit à la concurrence entre langue, peuple, nation et, non pas comme on le dit souvent, à leur confusion.

Pour montrer comment s'est effectué l'échec du dispositif européen comme mythe de la circulation européenne, nous aimerions nous arrêter à l'étude d'un exemple éclairant les impasses internes au monde mental de l'indo-européanisme.

L'incorporation de la question de l'origine de la langue parlée par les Tsiganes (que l'on appellera par commodité en dépit de ses multiples variantes le « romani cib », la langue romani) à la question indo-européenne fournit un

8. CRÉPON, 2000, p. 15.

9. OlENDER, 1989. 
exemple « chimiquement pur » des impasses indo-européennes. Des conditions anciennes de migrations multiformes et mal connues ne permettent pas encore à l'heure actuelle de procéder à une reconstitution vérifiée du destin collectif d'un peuple aux contours historiques ${ }^{10}$ flous, à partir d'un foyer originel de dispersion, que les linguistes s'accordent, pour leur part, à situer dans l'Inde du Nord ${ }^{11}$.

Dès l'époque de la Renaissance, dans un dispositif d'échanges savants étendus à l'Europe par la concomitance des premières incursions des « ducs et comtes de petite Égypte » vers 1450-1550, le nom des Bohémiens, Zigeuner, Gitanos, Égyptiens, Cigani venait sous la plume du triptyque savant de l'époque classique par des mentions incidentes et courtes. Les érudits cherchaient l'origine géographique et donc morale d'un peuple dont on admettait les caractéristiques culturelles, assez méprisables sans doute dans l'optique du temps, mais réelles. La langue, la promiscuité familiale, l'étonnante résistance physique d'un troupeau humain sauvage, le refus de la civilisation que le contact avec la société aurait dû provoquer, le mépris du bien d'autrui, la pratique de la divination et des danses « lascives », la connaissance des chevaux, l'habitude de manger des « nourritures infâmes », charognes d'animaux vite dépecés, et, last but not least, l'hypothèse formulée par certains de l'anthropophagie, tout cet ensemble de traits culturels avaient de quoi dérouter les savants et les curieux. De ce constat commun, singulièrement dévalorisant, les érudits dégageaient deux types de réflexion. L'usage d'une véritable langue, nettement distinguée d'un « jargon », supposait un degré de civilisation que venaient aussitôt démentir les pratiques repoussantes d'une peuplade sauvage. Comment expliquer la persistance au sein même de l'Europe civilisée de gens assez furieux pour refuser la contagion de civilité qu'implique par nature le contact avec celui qui vous est supérieur? De quelle nature était donc la résistance que les Tsiganes manifestaient dans leur refus des marques de la civilisation et d'élévation morale ? Comment expliquer leur volonté à se complaire dans l'abjection ? On comprend mieux que les observateurs les mieux intentionnés n'aient proposé comme solution à ce paradoxe que d'admettre l'origine indienne de la langue, mais de l'associer à une caste inférieure. On conciliait ainsi raisonnablement les contraires, le caractère de haute civilisation que représente la possession, l'usage et le maintien d'une

10. De fait, la carte des pérégrinations bohémiennes proposée à la lecture n'est que le résultat d'une reconstitution partielle de mentions de chroniqueurs, sorties de leur contexte et mises bout à bout. Rien ne prouve, en effet, que ces textes, souvent tronqués, évoquent la trace d'un peuple identique ; et même si l'on voulait retenir le principe de la continuité anthropologique entre l'origine indienne et la dispersion européenne, il resterait encore à dater les étapes de la dispersion et à en expliquer les raisons, voir Primo congresso di etnografia italiana, Roma, 19-24 Ottobre 1911, brevi cenni sullo studio della Tsiganologia in Italia, Relazione del Prof. Adriano Colocci, plaquette conservée à la bibliothèque municipale de Trieste, Lascito Hortis 6442. L'ouvrage de CoLOCCI, 1889, a été réédité à Bologne en 1971.

11. PredARI, 1841, chap. I, « Dell'origine dei Zingari ». 
langue véritable et originale dans un milieu étranger et l'inexistence, pire encore, le mépris de toute forme de vie civilisée chez les Bohémiens ${ }^{12}$. Ainsi, au XVIII ${ }^{\mathrm{e}}$ siècle, les Tsiganes connurent une incorporation savante à éclipses marquée par le mépris de l'objet-langue qui leur était propre et qu'il ne fallait pas confondre avec l'argot (Rotwelsch). La naissance et l'évolution de la tsiganologie ont été étroitement liées au contexte culturel de son développement. L'étude des Tsiganes est demeurée une activité marginale qui n'a pas connu, même dans le domaine linguistique, d'institutionnalisation véritable. Au début d'un ouvrage tout entier consacré aux Tsiganes, un auteur anglais, John Hoyland, affirmait, en 1816 : «The origin of this people has been a subject of inquiry for more than three hundred years. Many persons have been anxious to discover who these gusts were, that, unknown and uninvited, came into Europe in the fifteenth century, and have chosen ever since to continue in this quarter of the globe. » Il citait comme référence Heinrich M. G. Grellmann «translated a few years since, by the late M. Raper, Esq. F.R.S., \& A.S. », et il ajoutait: «He has, however, to acknowledge indebted to various others intelligent authors, whose writing will be noticed in the course of the work ${ }^{13}$. »

Pourtant la question des origines a passionné très tôt les historiens et les linguistes lancés sur la piste indo-européenne. De grands noms de la linguistique historique ont exploité les données lexicographiques rassemblées dès le $\mathrm{XVI}^{\mathrm{e}}$ siècle, les ont enrichies et utilisées dans leurs recherches sur la classification des langues, ou dans les tentatives de reconstitution de la langue originelle indo-européenne. En fait, l'identification même scientifiquement prouvée de la parenté entre la langue tsigane et les langues indiennes n'éclaircissait pas pour autant le mystère des migrations bohémiennes.

La langue romani a donc été reconnue comme d'origine indienne depuis l'âge des Lumières. Mais ce «pedigree » linguistique, à forte valeur ajoutée, n'entraîna pas pour autant l'institutionnalisation des études historiques et linguistiques. Les philologues soucieux de connaître cette langue - fort peu nombreux au demeurant mais étonnants polyglottes - ont été influencés par le

12. Recherches historiques sur le peuple nomade appelé en France bohémien et en Allemagne Zigeuner, avec un vocabulaire comparatif des langues indienne et bohémienne, traduites de l'allemand, de M. Grellmann, par M. le B. de B..., Paris, chez Belin et la veuve Duchesne, 1787, p. 48. Ce texte reprend celui de Grellman, 1783. Voir Willems, 1997. Le baron Jean-Nicolas-Étienne de Bock, lieutenant des maréchaux à Thionville, voyageur et correspondant du Journal encyclopédique, résumait l'opinion du temps. Les Tsiganes venaient selon lui de la caste inférieure des Sudders et, conviant Hérodote, il mentionnait que déjà celui-ci « fournit la preuve que les Sudders ne sont pas moins adonnés à l'anthropophagie que les Bohémiens ». À qui eut pu douter de la valeur d'un témoignage aussi ancien, l'auteur opposait : «Quelqu'ancien que soit son témoignage, il ne laisse pas d'être d'un grand poids, les mœurs et les coutumes, dans ces contrées éloignées, ne changeant jamais. »

13. HoYland, 1816, introd., p. IV et v. 
contexte scientifique de leur époque. Il importait alors de classer la distribution des langues, afin d'étayer la thèse des rameaux indo-européens. Ils recueillirent des vocabulaires quand ils ne se contentaient pas de recopier les listes de mots parus dans des ouvrages antérieurs et ils les confrontèrent au vocabulaire de langues indiennes. Ensuite, ils distribuèrent les dialectes romani selon leur degré supposé de pureté par rapport à une nomenclature idéale, et distribuaient les bons et les mauvais points aux tribus rencontrées.

Les Anglais, en particulier, ont voulu faire reconnaître la priorité de Jacob Bryant. Le 3 février 1785, Joseph Banks, qui fut président de la Royal Society, communiquait à la Society of Antiquaries de Londres une lettre de William Marsden qui concluait à l'origine indienne de la langue tsigane. William Marsden (1754-1836) était un numismate, orientaliste et linguiste irlandais ${ }^{14}$. Le docteur John Douglas, qui assistait à la séance, se souvint de son ami Jacob Bryant. Ce dernier transmit à la société ses travaux, qui furent présentés à la réunion suivante le 7 avril 1785. La même année, les travaux de Marsden et de Bryant furent publiés dans Archeologia et dans l'Annual Register de Dodsley ${ }^{15}$.

En 1911, le linguiste et tsiganologue anglais John Sampson, prétendait que son compatriote avait déjà démontré depuis 1776 l'origine indienne des Tsiganes ${ }^{16}$. Sampson fut l'auteur d'une étude classique sur le dialecte du pays de Galles ${ }^{17}$ qui montrait la persistance du fond indien dans le vocabulaire des Tsiganes de cette région encore au $\mathrm{XX}^{\mathrm{e}}$ siècle. Il appartint aussi aux Anglais de devenir les initiateurs de la Gypsy Lore Society et du Journal of the Gypsy Lore Society. Cette collection, commencée en 1888, rassemble le corpus de tout ce qui concerne le monde tsigane dans les différents continents ${ }^{18}$.

À la fin du XVII ${ }^{\mathrm{e}}$ siècle, l'hypothèse de l'origine indienne des Tsiganes avait été défendue simultanément par plusieurs auteurs. L'un d'entre eux, Jacob Carl Christoph Rüdiger est souvent cité comme l'un des premiers ${ }^{19}$. Ses recherches furent déterminées par la rencontre fortuite effectuée par un Hongrois et

14. Il devait, en 1813, attirer l'attention sur certaines ressemblances lexicales entre les langues du Congo et celles de la côte orientale de l'Afrique, voir Le Langage, sous la dir. d'André Martinet, Paris, Gallimard (Encyclopédie de la Pléiade), 1968, p. 1392.

15. BRYANT, 1785, et MARSDEN, 1785.

16. SAMPSON, 1907 et 1911, ainsi que TuRnER, 1927.

17. SAMPSON, 1926. La langue romani du pays de Galles a pris environ cent cinquante mots à l'anglais, trois douzaines à la langue galloise. On trouve cinq cent dix-huit mots d'origine indienne, alors que le total des emprunts allant de l'iranien au gallois ne se monte qu'à quatre cent trente. Ce dialecte est donc «l'une des langues indiennes les plus profondément étudiées », remarquait Jules Bloch, le grand indianiste, professeur de langue romani à l'École des langues orientales à Paris.

18. BURTON, 1898, part I : « Notes on Modern studies of “chinganology” », p. 136-201; part II : « Topographical notes on the Gypsies and Jats », p. 202-285.

19. RÜDIGER, 1782-1793, voir Stück I, p. 37-84 : «Von der Sprache und Herkunft der Zigeuner aus Indien. » 
des étudiants hindous. En 1763, le Hongrois Istvan Valyi rencontra en effet des étudiants venus de la côte de Malabar en Europe, et il constata les ressemblances de leur langue avec celle des Tsiganes. Les Tsiganes comprenaient assez bien les visiteurs que Rüdiger s'était empressé de leur présenter. Rüdiger confirma, en 1782, les constatations de Valyi.

Le grand linguiste August Friedrich Pott exerça un rôle important dans le développement des études indo-européennes. Il fit partie de la génération des «néogrammairiens » située entre Jakob Grimm et Franz Bopp, l'initiateur de la grammaire indo-européenne. Dès 1833, il avait eu l'idée d'utiliser les travaux antérieurs pour étudier l'étymologie dans les langues indo-européennes. Il entreprit le travail «inverse » de celui des fondateurs, « lequel consiste à [se servir] de leurs découvertes pour "redescendre" dans l'étude des langues particulières, dont ils éclairent l'origine ${ }^{20} »$. Pott voyait à l'intérieur de la famille indo-européenne des affinités particulières entre le latin et le grec, entre les langues baltiques et slaves. Par ailleurs, la connaissance désormais possible en Europe du sanskrit donnait la clé de l'iranien avestique ce qui, du même coup, révélait l'étroite parenté des familles indienne et iranienne. Pott publia en 1844 et 1845 une somme en deux volumes: Die Zigeuner in Europa und Asien ${ }^{21}$. Dans l'introduction, il résumait ainsi ses résultats. En premier lieu, les dialectes tsiganes de tous les pays montraient, malgré l'influence d'idiomes locaux, une homogénéité frappante. En second lieu, on ne pouvait reconnaître dans les dialectes tsiganes des langages populaires particuliers : la langue est différente de l'argot (Gaunersprache) avec lequel elle a été souvent confondue. Enfin, cette langue dérivait de façon irréfutable non pas de la langue égyptienne, mais des idiomes populaires de l'Inde du Nord et, malgré sa singulière bâtardise, elle pouvait se vanter d'appartenir au fier sanskrit.

Chose curieuse, Pott ne s'était adonné à l'étude de la langue tsigane que par hasard, en utilisant les notes laissées par son ami, spécialiste de sanskrit, Peter von Bohlen, qui passa ses dernières années à Halle ${ }^{22}$. Pott ne parla qu'une ou deux fois dans sa vie avec quelques Tsiganes et il se garda bien de multiplier les occasions de rassembler un matériau lexicographique direct. À ce propos, Francis Hindes Groome raconte l'anecdote suivante dans son livre In Gypsy tents, paru à Édimbourg en $1880^{23}$ :

«Je fis ma première visite au professeur Pott, qui m'affirma qu'il ne parla qu'une fois avec des Tsiganes quelque part à proximité de Londres. Alors je lui demandai si les Tsiganes ne venaient jamais à Halle et il me répondit que

20. SERGENT, 1995, p. 32.

21. Pотт, 1844-1845. La réimpression a paru en 1964, à Leipzig, au Zentralantiquariat. Voir aussi MORDTMANn et POTT, 1870.

22. Mode et WÖLFFLING, 1968.

23. Groome, 1880. Trad. d'Henriette Asséo. 
non. Là-dessus je pris congé de lui. À peine m'étais-je éloigné de 200 yards de sa maison que j'aperçus un chariot squelettique, traîné par deux petits chevaux. Dans le chariot était assise une femme qui fumait une grosse pipe noire et autour du chariot arrêté devant l'auberge, jouaient trois ou quatre enfants presque nus. Lorsque je pénétrai dans l'auberge, je vis deux Tsiganes attablés mangeant une soupe et buvant de la bière. Je les saluais en disant "Latcho divvus" (bonjour), eux ne parurent pas le moins du monde surpris, car ils étaient des messieurs qui avaient beaucoup voyagé. Ils avaient quitté la Hongrie depuis trois ans et avaient vécu en France et en Allemagne. Ils parlaient couramment le français et l'anglais. Nous nous entretînmes de différentes choses et, je me souviens, nous comparâmes nos passeports... Dans chaque localité où ils arrivaient, ils devaient d'abord se rendre à la police, qui leur indiquait les endroits où ils pouvaient stationner. Ils avaient le droit d'y rester trois jours sans être inquiétés par personne [...]. »

Ces résultats furent incorporés dans la vaste enquête menée dans la décade suivante par le grand philologue Franz Miklosich. Il réunit, à son tour, un important matériel lexicographique et il essaya de circonscrire avec exactitude la patrie primitive des Tsiganes ${ }^{24}$. Miklosich ne s'en tint pas à la parenté linguistique ; il espérait reconstituer les routes de migrations à partir du foyer indien primitif. Il étudia, à cet effet, les emprunts lexicographiques effectués au cours de ces migrations. L'itinéraire le plus plausible passait selon lui par la Perse, l'Arménie et la Grèce.

Dès le début du XIX ${ }^{\mathrm{e}}$ siècle, on était donc parvenu à répartir les principales langues d'Europe et d'Asie en ensembles dans lesquels on distinguait des sous-groupes. Cette classification n'était pas la seule en usage. Si Franz Bopp publia en 1816 son étude comparée sur la conjugaison du sanskrit, si Rasmus Rask écrivit en 1818, un mémoire sur la « famille » finno-ougrienne, August von Schlegel proposait, la même année, un classement tout différent : en se fondant sur l'étude des traits caractéristiques des langues et non de leur histoire, il distingua le type analytique, illustré par le chinois, le type synthétique, latin ou grec, et le type agglutinant, turc ou swahili. Dans la somme que constitua l'Indische Bibliothek ${ }^{25}$, il présentait les Tsiganes et leur langage. Graziado Isaïa Ascoli a produit aussi des Zigeunerisches ${ }^{26}$. Fondateur de l'Archivio glottologico italiano, il s'était penché sur la question des « substrats 27 », c'est-à-dire de restes de langues qui ont pu être parlées avant l'extension du latin et des autres

24. Franz von Miklosich (1813-1891), le linguiste slovène, appliqua la méthode comparative aux langues slaves et un premier bilan de ses recherches fut donné par le Compendium d'August Schleicher, voir PasPati, 1870.

25. SCHLEGEL, 1823 et 1824 . Les Tsiganes et leur langage sont étudiés dans le volume I, p. 427-430.

26. AsCOLI, 1865.

27. SERGENT, 1995, p. 44. 
langues italiques. Ce type de travail devait mobiliser l'énergie d'un grand nombre de linguistes du XIX ${ }^{\mathrm{e}}$ siècle. Enfin, la recherche sur la langue tsigane s'honora des travaux fort sérieux de l'archiduc Joseph, cousin de l'empereur François-Joseph ${ }^{28}$.

La réception de cet ouvrage dans le milieu tsigane mérite d'être exposée ${ }^{29}$ : l'Archiduc avait envoyé son livre, avec un autographe au chef des Tsiganes de Besztercze, le musicien György Karoly. Or, lui et ses compagnons avaient essayé de parler avec des Tsiganes turcs et à travers les mots qui semblaient pourtant les mêmes, ils n'avaient pu saisir le sens général de la conversation. C'était la faute de la grammaire de l'Archiduc, disait-il, car dans ce livre, il avait appris que les Tsiganes parlaient différents dialectes !

En France, la dissertation de Jacques Gråbert de Hemsö ne reçut pas l'écho espéré30 ${ }^{3}$ L'auteur était le vice-agent de commerce du roi de Suède, correspondant des académies de Stockholm et de Turin et aspirait à devenir correspondant de l'Institut impérial de France, sa correspondance en faisant foi. Il devait se faire connaître, outre ses compétences en arabe, par un mémoire publié en 1822 sur La Scandinavie vengée de l'accusation d'avoir produit les peuples barbares qui détruisirent l'Empire de Rome ${ }^{31}$. Il adressa donc un mémoire sur les Bohémiens et un autre sur les Samaritains à Sylvestre de Sacy.

Son mémoire sur les Bohémiens fut lu à la séance du 5 mai 1813 à Turin et la même année 1813, il fut nommé correspondant de l'Institut impérial de France, honneur dont il remercia longuement Sylvestre de Sacy ${ }^{32}$. Son acharnement à obtenir une reconnaissance sociale du monde savant ne fit pas rentrer les lettres tsiganologiques dans le cercle fermé des correspondants de l'Institut et de la Société royale des antiquaires de France. George Depping fit un rapport sur sa dissertation en affectant de se saisir de l'intérêt érudit du sujet pour le dévaluer ${ }^{33}$ :

«Depuis que l'étude des langues a mis les savants dans la bonne route pour découvrir l'origine des peuples, on a commencé aussi à acquérir des notions plus saines sur les Bohémiens, peuple singulier qui parcourt toute l'Europe, ne s'attache à aucun pays, et préfère la vie misérable à tous les avantages de la vie sociale dans les pays civilisés ${ }^{34}$. »

28. Czigány nyelvtan Romano csibàkero sziklaribe vita Jözsef Föherczeg, Budapest, Kiadja a Magyar Tudomá nyos Akadémia, 1888.

29. PENNELL, 1893, p. 176.

30. GRÅBERT DE HEMSÖ, 1813.

31. Sa nièce et fille adoptive, Jacqueline Eleonore Gråberg, écrivit une notice historique sur sa vie, parue à Florence en 1831.

32. Correspondance générale de Sylvestre de Sacy, ms 2377, pièce 130, lettre du 29 novembre 1813, pour publication dans le Magasin encyclopédique ou dans les Annales de MalteBrun, Paris, Bibliothèque de l'Institut.

33. DePPING, 1817.

34. DepPING, 1817, p. 246. 
Comme l'avaient prédit Tocqueville et Renan, l'ouvrage de Joseph Arthur comte de Gobineau fut bien reçu dans les pays germaniques. Son érudition et sa connaissance réelle du dossier indo-européen y expliquent le succès de l'Essai sur l'inégalité des races humaines (1853-1855). Mais il demeurait l'adepte de la théorie asiatique des indo-européens : les Aryens étaient originellement une race pure, blanche et blonde, originaire de l'Iran, mais cette race primitive ne pouvait se retrouver car elle s'était mêlée, à des degrés divers, selon les peuples avec les Sémites. La hiérarchisation proposée par Gobineau partait du diagnostic de dégénérescence plus ou moins avancée des sociétés selon le degré de métissage. Notre linguiste Pott rédigea aussitôt un livre qui voulait confirmer entièrement les idées de Gobineau, intitulé L'Inégalité des races humaines, surtout du point de vue de la science philosophique, en considération de l'ouvrage du comte de Gobineau portant le même titre et publié en $1856^{35}$.

Curieusement, le maintien des positions de Gobineau sur l'existence du berceau asiatique des Indo-Européens avait stimulé son intérêt pour l'étude de la langue romani. Prosper Mérimée, alors à la recherche de glossaire de bonne qualité et de matériel lexicographique, en témoigne dans sa correspondance ${ }^{36}$ dont l'extrait suivant donne le ton général : «[...] je vous envoie ci-joint quelques mots bohémiens qui sont en Europe d'un usage général parmi les tribus errantes depuis la Russie jusqu'à l'Espagne. Peut-être vos ??looties [sic] de la Perse les entendront-ils ? » De Téhéran, Gobineau s'interrogeait sur la parenté entre les Ghadjars et «nos Bohémiens d'Europe ». Or, Mérimée était par ailleurs en correspondance avec Francisque Michel dont il admirait l'Histoire des races maudites ${ }^{37}$. Il en attendait aussi l'envoi de matériaux linguistiques : « [...] pour compléter mes études sur la langue romani, j'attends que vous nous donniez un troisième volume de vos Races maudites ${ }^{38}$. »

L'anthropologie physique cherchait à établir scientifiquement un système de classement des êtres humains semblable à celui proposé par les sciences naturelles pour les animaux. C'est ainsi que le concept de « race » se chargea progressivement d'un contenu anthropologique. Au début du XIX ${ }^{\mathrm{e}}$ siècle, il avait encore un sens vague, proche de celui de «famille », de « descendance » et même de «nation» au sens ancien du terme. Il devint le terme spécifique pour désigner des entités à l'intérieur de l'espèce humaine, distinguées par les mensurations des os, surtout du crâne, et secondairement, par la couleur de la peau, des cheveux, des yeux. C'est aussi en toute bonne foi que les adeptes de

35. SERGENT, 1995, p. 40.

36. MÉrIMÉE, 1953, p. 431-432. Voir aussi, ibid., p. 539-540 : Lettre au comte de Gobineau du 9 février 1855, et lettre du 20 mars 1855.

37. Né à Lyon en 1809, Francisque Michel a édité une trentaine d'ouvrages de littératures médiévales anglaises et françaises. Sa thèse en histoire et en philologie présentée pour le doctorat ès lettres en 1847 fut publiée sous le titre Histoire des races maudites.

38. MÉRIMÉE, 1930. 
l'anthropologie physique exercèrent leurs talents sur les mensurations des crânes tsiganes.

Ainsi le professeur Abel Hovelacque se félicitait de l'invention du craniophore du professeur Paul Topinard - une remarquable invention recommandée par Paul Broca qui réduisait les vicissitudes de l'angle facial - pour présenter devant la Société d'anthropologie, en 1874, ses conclusions portant sur une série d'une « richesse particulière de douze crânes tsiganes, versés aux collections du Muséum ${ }^{39}$ ». Cinq d'entre eux, originaires de Roumanie et de Transylvanie, avaient été envoyés par le prince George Cantacuzène. Il restait à mesurer la capacité de la boîte crânienne. Isidore Kopernicki, qui reçut pour ses travaux le prix Godart de la Société d'anthropologie, avait cubé les siens avec succès en utilisant du millet bien sec. L'opération refaite par Hovelacque lui fit conclure que « le crâne tsigane est donc relativement petit ».

L'Europe scientifique, prise au piège de son propre dispositif indianiste, admit l'incorporation des Tsiganes à l'aryanité. Elle prit soin de séparer les Tsiganes idéalement purs et introuvables de l'immense masse de «métis », sortes de composés de castes inférieures qui campaient aux abords des cités. Quelquefois, sous la crasse, l'observateur reconnaissait à ces aryens un « fier profil hellène » (sic). Par un étrange mimétisme, le Tsigane imaginaire se mit à ressembler au tsiganologue ; et tel le docteur Jeckill dédoublé en mister Hyde, il était tour à tour paré de toutes les vertus de l'innocence de la Nature, ou dépravé et affublé des traits d'une sauvage inhumanité.

L'exemple des Tsiganes illustre l'échec de la construction indianiste classique comme mythologie transeuropéenne puisque la possession d'une langue aryenne, maintenue vivante en dépit des vicissitudes de l'errance, ne garantissait nullement d'être maintenu au panthéon de la race supérieure.

À partir de la fin du XIX ${ }^{\mathrm{e}}$ siècle, une thèse nouvelle, autrement plus pernicieuse, celle de la « race nordique », prétendait que le berceau de la race aryenne était d'origine européenne et non asiatique ${ }^{40}$. C'était en Europe du Nord que se trouvait, selon les théoriciens de la « race nordique », le réservoir primitif et menacé de la race blanche pure caractérisée par une grande taille, une certaine dolicocéphalie et une pigmentation claire. Ces constructions fantasmagoriques recoupèrent les relations entre la linguistique comparée et l'anthropologie physique pour conduire à la « science de la race » de triste mémoire. Mais il n'existe pas de déterminisme direct entre l'affirmation de l'utopie européenne de la langue commune et le succès des thèses raciologiques. C'est le repli nordique qui a rendu ces thèses criminelles par le déplacement géographique de l'expertise anthropologique européenne d'un berceau mythique avestique à

39. Hovelacque, 1874.

40. CONTE et ESSNER, 1995. 
une territorialisation internaliste de la politique de la race. Combinée avec une classification fondée sur la hiérarchisation et l'inégalité biologique, leur puissance de conviction politique devrait pénétrer bien au-delà du cercle restreint et virulent des ultranationalistes allemands ${ }^{41}$.

Les arguties pseudo-scientifiques des stratèges de la race firent perdre aux Tsiganes leur dernier crédit linguistique. Établis en solides dynasties familiales sur les terres germaniques depuis la fin $d u X V^{e}$ siècle et surtout le $\mathrm{XVI}^{\mathrm{e}}$ siècle, les Zigeuner n'avaient choisi l'enracinement que pour mieux corrompre le sang allemand, opérant pernicieusement cette déliquescence du sang nordique en une cinquième colonne du métissage prolifique.

Les penseurs de la race décidèrent alors d'abandonner la thèse classique de l'origine indienne des Tsiganes et l'idée d'un peuple unique pour s'orienter vers celle d'un métissage social, faisant, non sans arrière-pensée, des Bohémiens un « composé de toutes les nations ». La langue n'était à leurs yeux plus qu'un « jargon », une langue secrète des bas-fonds, une sorte de Rotwelsch ${ }^{42}$. Partant de l'argument linguistique ou de l'argument social, ils prônaient la même politique d'exclusion à l'égard de la population incriminée. La volonté marquée de procéder à l'extirpation des Tsiganes de la nation biologique allemande fut à l'ordre du jour bien avant l'arrivée au pouvoir des nazis ${ }^{43}$.

Ainsi, le parcours en tout sens autorisé d'un espace commun, la cartographie maîtrisée de la terre appréhendée avec rigueur et gourmandise à « vol d'oiseau », la simplification des questions religieuses n' auront pas suffi, au XIXe comme au $\mathrm{XX}^{\mathrm{e}}$ siècle, à formuler la civilisation commune. Un goût fatal pour la Gemütlichkeit a existé dans les esprits les plus rebelles et la modernité, au nom de la guerre purificatrice, a détruit le compromis social instable, et chèrement acquis par les luttes sociales, de la démocratisation et de la modernisation des sociétés européennes.

De l'utopie de la langue, il n'est rien resté ou presque. Peut-être, aux marges de l'Europe, certains érudits excentrés, sortes de cosmopolites des confins, pratiquaient encore avec confiance la théorie de la traductabilité. Un certain James Pincherle a publié, à Trieste, en 1875, un fascicule dédié, précisément, au

41. LindeRMANN, 2001, p. 221-281.

42. GIRTLER, 1998.

43. Hans K. F. Günther devint «l'oracle anthropologique du national-socialisme ». En 1944, Josef Mengele lançait son programme propre sur des enfants jumeaux, juifs et tsiganes. Le professeur Ottmar von Vershuer suit celui-ci avec la plus grande attention en encourageant son assistant et « en parle au camp de concentration d'Auschwitz en tant que Hauptsturmführer et médecin du camp », voir Müller-Hill, 1989, p. 78-79. On ne peut prétendre que l'opinion scientifique n'ait pas été informée des actions d'euthanasie menées en 1934 en Allemagne. Voir SCHREIBER, 1935. 
consul de Grande-Bretagne dans cette ville, sir Richard Burton ${ }^{44}$. Il y donnait la traduction du Cantique des cantiques dans la langue tsigane, car il avait remarqué que ce texte avait été traduit dans le plus de langues possibles, même en basque par Louis-Lucien Bonaparte ou dans le dialecte des mineurs du Northumberland, mais jamais dans l'idioma zingaresco. Il écrivit aussi une adaptation et un prolongement du Voyage sentimental (1768) de Lawrence Sterne et il s'exprimait en judéo-espagnol.

Un siècle plus tard, le romani est encore parlé par les Tsiganes d'Europe, mais l'univers du romipen - la manière tsigane de voir le monde - a perdu de sa puissance visionnaire, et il ne reste rien du judéo-espagnol quand Marcel Cohen écrit à Antonio Saura :

«Cher Antonio, je voulais t'écrire en djudyo avant que s'éteigne tout à fait la langue de mes ancêtres. Tu n'imagines pas, Antonio, ce qu'est l'agonie d'une langue. C'est un peu comme se retrouver seul dans le silence ? C'est se sentir sikileoso [anxieux, oppressé, en turc] sans comprendre pourquoi ${ }^{45}$. »

Utopie de la langue doublement perdue : ce fut surtout en français que les Juifs séfarades d'Europe exprimèrent les derniers soupirs de leur asphyxie collective. Le «tohu-bohu» de l'expérience concentrationnaire, maelström nauséabond rassemblant avant la mort certaine en certains lieux assignés une multitude de personnes venant de tous les coins de l'Europe parlant toutes sortes de langues, fut bien perçu comme l'un des éléments qui empêchait la permanence de soi ${ }^{46}$. Primo Levi oppose à Babel l'angoisse inscrite en chacun de nous du «tohu-bohu, de l'univers désert et vide, écrasé sous l'esprit de Dieu, mais dont l'esprit de l'homme est absent : ou pas encore né ou déjà éteint $^{47} \gg$.

\section{LES DANGERS DE L'ETHNOPOLITIQUE}

Le remembrement ethnique de l'Europe, voulu par les nazis pendant la Deuxième Guerre mondiale, dans sa dimension apocalyptique, a été provoqué par l'échec des plans de recolonisation à l'Est. Aucun de ces plans ne pouvait satisfaire, en théorie et en pratique, le nouvel horizon d'attente dévoyé de la

44. Il Cantico dei cantici di Salomone per la prima volta tradotto dal testo italiano in fronte nell' idioma zingaresco (indo-orientale) studio di James Pincherle, Trieste, Giovanni Balestra e c. (Opusculiana Alberto Boccardi, 110), 1875.

45. COHEN, 1997, p. 9.

46. POLlaK, 2000, p. 11.

47. LEVI, 1989, p. 84. La langue allemande du camp est une variante de la langue du III ${ }^{\mathrm{e}}$ Reich, voir KLEMPERER, 1996. 
transfrontière germano-européenne ${ }^{48}$. Avant 1914, les pangermanistes qui se penchaient sur le problème de la question des confins n'envisageaient que l'expulsion, le territoire sans les hommes, Land ohne Menschen, comme solution à la gestion différentielle des populations. Ils pensaient que la politique d'évacuation qu'ils préconisaient constituerait déjà une rupture inouïe dans le droit et la culture internationale puisque, jusqu'en septembre 1914, Heinrich Class se plaignait de la difficulté à faire «avaler » aux décideurs l'idée que la « libération des territoires » supposait d'en expulser les populations ${ }^{49}$.

Aussi brutale que semble être la formulation, celle-ci se situait aux antipodes de la «solution finale ». Mais la «culture de l'evak», de l'expulsion forcée, marque définitivement l'échec du mythe indo-européen. La mythologie indianiste voulait établir la classification des peuples par la proximité linguistique. À l'opposé de son postulat de départ, la « distance ethnique », imposée par le mythe de la race nordique, fonde la survie des peuples. Cette politique est intraeuropéenne. Désormais, l'Européen n'est plus le maître d'une construction mentale homogénéisante ; contournant le problème de la nation, les territoires flottants de la race imposeront le primat de l'ethnopolitique.

«Un langage montre de façons diverses qu'il porte en lui le germe de la dissolution », nous dit George Steiner, et il ajoute : «Qui a donné la mort à la langue allemande ? C'est là un point d'histoire fascinant et compliqué. Il commence avec un fait paradoxal ; l'allemand fut plus vivant avant que n'existât un État allemand unifié. [...]. Les maîtres de la prose et de la poésie allemande furent des hommes qui ne connurent pas le dynamisme de la conscience nationale prusso-germanique, tel qu'il s'est développé après la fondation de l'Allemagne moderne en 1870. Ils furent, comme Goethe, des citoyens de l'Europe, vivant dans des principautés trop étroites pour solliciter les émotions du nationalisme. Ou, comme Heine et Nietzsche, ils écrivaient hors d'Allemagne. Et cela est resté vrai pour le meilleur de la littérature allemande, même dans une période récente. Kafka écrivit à Prague, Rilke à Prague, Paris ou Duino ${ }^{50}$. »

L'échec intellectuel à penser l'intégration des Européens par la langue à une souche originelle commune a eu les plus graves conséquences. Car nous pensons que c'est l'échec de la formulation de la question indo-européenne comme une formulation mythique de la mobilité et de l'élasticité de la civilisation européenne dans ses multiples métamorphoses, qui entraîna les bourgeonnements

48. Ainsi s'explique le débat sur le sort des Tsiganes sous le nazisme dans l'historiographie récente du génocide depuis la parution de FRIEDLANDER, 1995.

49. Korinman, 1999, p. 59.

50. STEINER, 1969, p. 95 et 96. 
néfastes et non l'inverse. Et en particulier, les conséquences criminelles de son rapatriement territorial vers les espaces de la germanité de sang.

Il n'est resté de cette vaste entreprise langagière que l'idée d'une Europe promise à une inéluctable décadence physique qui la conduirait à l'abîme. On jeta aux orties l'espoir de concilier identité nationale et transeuropéanisme par la traduction des langues les unes dans les autres et de servir par cette entreprise la cohésion d'une morale universelle. Les peuples de l'Europe, affolés de se découvrir si divers et si variés, crurent qu'ils jouaient leur existence dans une concurrence de voisinage.

Ainsi fut radicalement transformée, à partir de 1907, la construction de l'ennemi de l'intérieur en Europe. Le complot de contre-société des milieux interlopes, la menace grandissante de la subversion sociale, la suspicion généralisée à l'égard de la criminalité de bande n'était plus une affaire sociale et nationale, un enjeu de police et d'interception à la frontière. Ce fut, en tous pays, au même moment, une menace étrangère et invisible. Agents du délitement biologique par le métissage, les ennemis de la civilisation européenne avaient changé de nature. Il n'est rien resté de la glorification ancienne du cosmopolitisme comme parcours éclairé de l'Europe de la civilité et de la politesse généalogique. La mobilité est associée à l'idée de décadence et le génie indompté s'est transformé en "gêneur public».

L'analyse de la mutation de l'Europe ne peut en rester à la description des enjeux politiques des négociations engagées en 1919. Le «moment Wilson» pouvait apporter avec le travail des experts de l'Enquiry un élément nouveau dans la reformulation de l'Europe comme espace transnational par la « labellisation » démocratique des nationalités métamorphosées en États-nations successeurs des Empires européens.

Pourtant, « l'innocence grammaticale » ne garantit pas toujours d'honnêtes discours. On peut associer, dans le passage du cosmopolitisme classique à une forme originale de néonationalisme mondialiste de l'entre-deux-guerres, des personnages qui présentent à la fois des idées communes sur la rénovation spirituelle de l'Europe et des obédiences politiques opposées sur la mission particulière de l'Allemagne. La tradition idéaliste sert de lien. Ainsi Walter Rathenau, le président du trust de l'électricité AEG, le ministre des affaires étrangères de la république de Weimar assassiné par les nationalistes, réclamait avant la Première Guerre une révolte radicale contre la mécanisation. La violence du conflit mondial avait confirmé ses thèses : la société devait disparaître devant une communauté organisée selon l'Esprit, d'où le capitalisme égoïste et le prolétariat dictatorial seraient également bannis. Ce serait alors un retour au « germanisme vrai ». Au total, une Allemagne moderne regermaniserait le continent et y créerait la « démocratie véritable », qu'il appelle le Volksstaat, 
l'État adapté à la vie substantielle du peuple ; Hans Günther avait apprécié ce texte 51 .

Les paradoxes de la paix de 1919, situés entre l'espoir d'une restauration du cosmopolitisme à l'échelle du droit international emprunté à Grotius et la reconnaissance démocratique de l'ethnopolitique, ont établi une Europe étrangement proche de nous. Les frontières de l'Europe se sont voulues à nouveau mentales et non impériales, lorsque la démocratie s'est mêlée de la langue des peuples par l'organisation des plébiscites. Les négociations de paix ont favorisé une course à la territorialisation des appartenances à géométrie variable, ouvrant la voie à la concurrence langagière des nationalités et à la surenchère politique.

La question d'Orient concentrait déjà toutes les composantes culturelles de l'indécision européenne. Édouard Driault évoque la légitimité du démantèlement de l'Empire ottoman par l'affirmation d'un droit d'ingérence qui brouillait, au nom des valeurs supérieures, la traditionnelle ligne d'affrontement entre Orient et Occident. Il évoquait en 1938, à propos de la paix en Méditerranée, la question de l'Arménie et écrit dans sa préface :

« J'ai écrit ma première question d'Orient en 1898 sous le coup de l'indignation produite dans le monde entier par les massacres d'Arménie : et tout de suite j'ai condamné à mort l'Empire ottoman coupable de tant de forfaits. Ce jugement a été appliqué dans les vingt ans qui ont suivi ; et l'exécution capitale a été le fruit de la Grande Guerre. Il se pourrait que ce fut historiquement son principal résultat ${ }^{52}$. 》

Le droit d'ingérence serait le terrain d'expérimentation du plus petit commun dénominateur territorial d'unité dite européenne.

Les Balkans furent aussi le terrain de la tentation du tout minoritaire; l'historien hongrois, Oscar Jaszi, cité par Mark Mazower, observait dans un essai de 1925, à propos de la montée irrésistible de l'idée nationale, que la Paix n'apporterait pas de solution au problème des nationalités tant que le principe de l'autodétermination ne serait pas mis en pratique pour chaque groupe ethnique. La construction de la Tchécoslovaquie et de la Yougoslavie pouvait être les formes alternatives d'États nations mais l'application du principe de nationalités demeurait dans ces régions un ferment de violence ${ }^{53}$.

51. VERMEIL, 1948 : il présente les «apôtres attardés de la Renaissance spirituelle », Hermann Keyserling, Walter Rathenau et Thomas Mann, p. 39-109; la note 1, p. 41, renvoie aux approbations par Hans F. K. GüNTHER des dangers de la dégermanisation contenues dans les Reflexionen (1908) de Rathenau, publiées dans Rassenkunde des deutschen Volkes, Munich, 1922, p. 460-461. Les éditions successives de Rassenkunde des deutschen Volkes ont été rassemblées in LUTZHÖFT, 1971.

52. Driault, 1938, préf., « Le nœud de la question d'Orient », p. v.

53. MAZOWER, 2000, p. 76. 
Mais, là encore, le déterminisme nationaliste n'existe pas. La Tchécoslovaquie avait suivi une voie originale. À une époque où la centralisation était considérée comme une idée tout à fait moderne, rationnelle et progressiste, l'État tchécoslovaque avait pris le fait national en considération par la médiation de la démocratie. Chaque individu, lors du recensement, devait, comme sous l'Empire, déclarer sa nationalité. Cette prise en compte n'était pas une formalité purement statistique, elle servait de base à la répartition des écoles, à l'établissement de la langue usuelle dans telle ou telle région, à la nomination des fonctionnaires en proportion de la population de chaque nationalité. Il n'y a pas de fatalité politique «puisque les années 1925-1933 sont celles de l'éphémère ralliement des Allemands passant du "négationnisme" revanchard, à "l'activisme" non seulement loyaliste mais aussi participatif ${ }^{54}$ ». Mais la culture des Juifs tchèques de langue allemande ne fut pas mieux reconnue par les Allemands que par les Tchèques.

Même dans le cadre d'une démocratie pacifiée, le choix de la nationalité peut-il faire partie des « libres choix ${ }^{55}$ »?

Le territoire européen a pu être labouré de toutes parts, par un ensevelissement de corps pendant la Première Guerre mondiale, par les anéantissements familiaux de la Seconde Guerre mondiale, par les transportations exceptionnelles de l'Est, sans inscription dans la conscience collective d'un imaginaire collectif de la mobilité de masse.

La confrontation interdite entre la territorialisation de la mémoire nationale autorisée et les éclats rémanents de mémoires familiales entraînait un sentiment d'appartenance flottant, suffisant pour assurer la mise en route de millions d'Européens vers les nouveaux mondes ou les territoires de la colonisation, mais insuffisants pour construire une identité européenne. Ainsi s'explique la dévalorisation du cosmopolitisme :

« Notre siècle a pu sur tous ces points affronter le pire et le meilleur et se colleter sans succès à deux défis inscrits dans le patrimoine des Lumières : celui kantien, mais déjà proclamé par Montesquieu et Adam Smith que le cosmopolitisme, le commerce doux et prospère, assureront la paix du monde ; celui des athlètes de la civilité et de la sociabilité victorieuse des violences, dont plus près de nous Norbert Elias théorisera les idées ${ }^{56}$. »

Notre réflexion sur les rapports entre la représentation du mouvement et l'identité mentale de l'Europe a été guidée par la volonté de renverser la proposition habituelle sur les rapports entre mobilité et constructions identitaires. À travers les siècles, l'Europe a connu en son sein toutes les formes de mobilités :

54. MERCIER, 2000, t. II, p. 312-313.

55. Kieval, 2000.

56. ROCHE, 1999, p. 11. 
des moines girovagues et des armées en campagne, des déplacements brutaux de populations issus des multiples partages ethniques, des royautés elles-mêmes nomades, des migrations provinciales jamais définitives, les exodes des « grandes guerres », sans compter le mouvement perpétuel des bateaux pour les Nouveaux Mondes. Pétrie par ces migrations multiples et enchevêtrées, marquée par le poids prépondérant jusqu'au XIX ${ }^{\mathrm{e}}$ siècle d'une mortalité collective très élevée, son histoire est pleine de peuples à la renommée éteinte dont le nom n'est même pas parvenu jusqu'à nous ${ }^{57}$.

Pour autant la capacité des populations européennes à se mouvoir en évitant tout à la fois l'oubli des origines et la ségrégation communautaire, dans des conditions de remise en route, volontaire ou contrainte, observées souvent à des siècles de distance, ne me semble pas, en effet, l'apanage exclusif des minorités migrantes « labélisées », mais le produit d'une disposition commune de la civilisation européenne, assumant dans un même mouvement la dispersion et l'enracinement, et formant le substrat d'une « culture de la circulation ».

Ainsi, l'un des aspects de la « grammaire cachée » du déplacement se trouverait niché dans les méandres de ce que j'appelle le «principe de circulation européen ». La construction des identités nationales et des frontières des États européens et les usages du principe des nationalités en auraient masqué jusqu'à l'existence. Ces procédures de refoulement ne disent rien de l'ampleur et de la variété des déplacements réels à travers l'Europe, mais elles ont focalisé sur les minorités migrantes la vision d'agents potentiels d'un démantèlement des ordres nationaux.

Aussi, on reste désarmé devant l'offensive séduisante des philosophes du renouveau cosmopolitique. «Entre, qui que tu sois » demeure un horizon inaccessible de l'hospitalité pure telle que la décrit Jacques Derrida ${ }^{58}$. À première vue, cette proposition ne paraît pas si éloignée du projet universaliste des Lumières : «Porte tes pas où bon te semble. » Mais l'arpentage éclairé de l'espace de civilité induisait cependant une séparation entre le dehors et le dedans. À l'exception de cas limites comme ceux des Juifs et des Tsiganes, l'étranger venait toujours de l'extérieur. Derrida renverse délibérément tout le dispositif historique européen de séparation mentale entre soi et les autres. Mais, si notre propos se vérifie quelque peu, le danger du refus de l'hospitalité ne viendrait pas du raidissement xénophobe, mais du délitement identitaire collectif. Qui, dans cette hypothèse, deviendrait l'otage de l'autre ? Dans le ventre mou d'un espace marchand, l'étranger rassasié s'y perdrait autant que son hôte.

57. DuCELLIER et al., 1992. Cet ouvrage étudie en particulier le cas des migrations albanaises provoquées par les guerres permanentes qu'entraîna, bien avant la conquête ottomane, l'éclatement de l'Empire byzantin.

58. DERRIDA, 1997. 
L'historien du passé-présent se trouve donc à la croisée des chemins. Il voit s'effriter sa légitimité nationale et académique. En même temps, il est convoqué par les cours de justice comme expert, une sorte de médecin légiste prié de sortir les cadavres des placards à heure dite. Il fut un temps plus glorieux (et plus dangereux) où il rêvait de conseiller les princes, le voici appelé au grand partage des dépouilles victimaires d'un siècle infâme.

Il est grand temps de partir « respirer à l'aise », comme le disait Marc Bloch, dans de plus vastes espaces européens, sans rien perdre de son identité et de considérer les enjeux d'une civilisation commune pour le meilleur et pour le pire.

Henriette AssÉo (octobre 2001). 


\section{LISTE DES RÉFÉRENCES}

Ascoli (Graziado Isaïa), 1865, Zigeunerisches. Von G. J. Ascoli. Besonder auch als Nachtrag zu dem Pott'schen Werke : "Die Zigeuner in Europa und Asien », Halle/ Londres/Turin-Florence, E. Heynemann/Williams \& Norgate/Hermann Loescher.

BALDENSPERGER (Fernand), 1940, Une vie parmi d'autres. Notes pour servir à l'histoire de notre temps, Paris, Louis Conard.

BASChET (Jérôme), 2001, «L'histoire face au présent perpétuel », in HARTOG et REVEL, dir., 2001, infra, p. 55-74.

BELl (David Avrom), 2002, «Le caractère national et l'imaginaire républicain au XVIII ${ }^{\mathrm{e}}$ siècle », Annales. Histoire, sciences sociales, juil.-août, 4, p. 867-888.

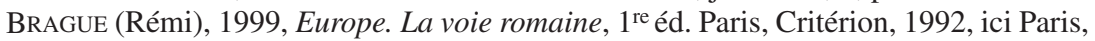
Gallimard (Folio essais).

BRYANT (Jacob), 1785, « Collections on the Zingara or Gypsey Language », Archaeologia (Londres), vol. VII, p. 387-394.

Burton (Richard Francis), 1898, The Jew, the Gypsy, and El Islam by the late captain Richard F. Burton, ed. with a preface and brief notes by Williams H. WILKINS, Londres, Hutchinson and Co.

CoHEN (Marcel), 1997, Lettre à Antonio Saura, trad. du judéo-espagnol, éd. bilingue, Paris, L'Échoppe.

Colocci (Adriano), 1889, Gli Zingari, storia di un populo errante, Turin, Ermanno Loescher, reprod. anastatique, Bologne, Forni editore, 1971.

CONTE (Édouard) et ESSNER (Cornélia), 1995, La Quête de la race. Une anthropologie du nazisme, Paris, Hachette.

CRÉPON (Marc), 2000, Le Malin génie des langues (Nietzsche, Heidegger, Rosenzweig), Paris, Vrin.

DePPING (George Bernard), 1817, «Rapport sur la dissertation de M. Graberg de Hemsoe, intitulée : Doutes et conjectures sur les Bohémiens et leur première apparition en Europe, etc. », Mémoires et dissertations sur les Antiquités nationales et étrangères publiés par la Société royale des antiquaires de France, t. I, Paris, Fournier, libraire, p. 246-250.

Derrida (Jacques), 1997, De l'hospitalité, Paris, Calmann-Lévy (Petite bibliothèque des idées).

DriaUlt (Édouard), 1938, La Question d'Orient, 1918-1937, la paix en Méditerranée, Paris, Félix Alcan (Bibliothèque d'histoire contemporaine).

DuCELlier (Alain) et al., 1992, Les Chemins de l'exil. Bouleversements de l'Est européen et migrations vers l'Ouest à la fin du Moyen Âge, Paris, Armand Colin.

FRIEDLANDER (Henry), 1995, The Origins of Nazi genocide from euthanasia to the final solution, Chapel Hill, NC, University of North Carolina.

GiRTLER (Roland), 1998, Rotwelsch. Die alte Sprache der Gauner, Dirnen und Vagabunden, Vienne, Böhlau. 
GrÅBERT DE Hemsö (Jacques), 1813, Doutes et conjectures sur les Bohémiens et leur première apparition en Europe. Extrait des Mémoires de l'Académie impériale des sciences, littérature et beaux-arts de Turin pour les années 1811-1812, Turin, chez Félix Galleti, imprimeur de l'Académie, t. XXI, sér. Littérature et Beaux-arts, 5, p. 17-40.

Grellman (Heinrich M. G.), 1783, Die Zigeuner. Ein historischer Versuch über die Lebensart und Verfassung, Sitten und Schicksale dieses Volks in Europa, nebst ihrem Ursprunge, Dessau-Leipzig, auf Kosten der Verlagskasse.

Groome (Francis Hindes), 1880, In Gipsy tents, Édimbourg, W. P. Nimmo \& Co.

Hartog (François) et Revel (Jacques), dir., 2001, Les Usages politiques du passé, Paris, Éditions de l'École des hautes études en sciences sociales (Enquête).

Hovelacque (Abel), 1874, «Sept crânes tsiganes », Revue d'anthropologie, vol. II, p. 161 et vol. III, p. 234.

HoYLAND (John), 1816, A historical survey of the customs, habit \& present State of the Gypsies; designed to develop the origin of this singular people, and to promote the amelioration of their condition, York, William Alexander.

KIEVAL (Hillel J.), 2000, Languages of community. The Jewish experience in the Czech Lands, Berkeley, CA, University of California Press.

KLEMPERER (Victor), 1996, LTI. La langue du III Reich. Carnets d'un philologue, trad. de l'allemand par Élizabeth GuILLOT, Paris, Albin Michel.

KorinMan (Michel), 1999, Deutschland über alles. Le pangermanisme, 1890-1945, Paris, Fayard (Pour une histoire du XX $\mathrm{XX}^{\mathrm{e}}$ siècle).

LeVI (Primo), 1989, Les Naufragés et les rescapés, quarante ans après Auschwitz, trad. de l'italien par André MAUGÉ, Paris, Gallimard (Arcades).

LiNDERMANN (Thomas), 2001, Les Doctrines darwiniennes et la guerre de 1914, Paris, Economica (Hautes études militaires).

LutZhöFt (Hans Jürgen), 1971, Der nordische Gedanke in Deutschland 1920-1940, Stuttgart, Klett.

MANCINI (Silvia), 1999, «Les civilisations comme "absolu esthétique". L'approche morphologique de la Mittel-Europa », Diogène, 186, p. 88-109.

MARSDEN (William), 1785, «Observations on the language of the people commonly called Gypsy. In a letter to sir Joseph Banks, with tables comparing the Gypsy and Hindustani languages », Archaeologia (Londres), vol. VII, p. 382-386.

Mazower (Mark), 2000, The Balkans. A short history, New York, NY, Modern Library.

MERCIER (Laurent), 2000, La Tchécoslovaquie des Français. Conceptions et représentations des Pays Tchèques et de la Slovaquie par les Français de la Troisième République, thèse de doctorat d'histoire, dir. par Jean-Jacques BECKER, université Paris X, 2 t.

MérimÉE (Prosper), 1930, Lettres à Francisque Michel, 1848-1870, texte établi et annoté avec une introd. par Pierre TRAHARD, in Euvres complètes de Prosper Mérimée, Paris, Honoré Champion.

MÉRIMÉE (P.), 1953, Correspondance générale, établie et annotée par Maurice PARTURIER, $2^{\mathrm{e}}$ sér., t. I : 1853-1855, Toulouse, Privat.

Milosz (Czeslaw), 1987, Témoignage de la poésie, trad. du polonais par Christophe JeZEWSKI et Dominique Autrand, Paris, Presses universitaires de France (Écriture).

Mode (Heinz Adolf) et WöLfFling (Siegfried), 1968, Zigeuner. Der Weg eines Volkes in Deutschland, Leipzig, Koehler \& Amelang. 
Mordtmann (Andreas) et PотT (August Friedrich), 1870, Zigeunerische Zeitschrift der deutschen morgenlichen Gesellschaft (Leipzig), vol. XXIV, p. 681-703.

MüLlER-HILl (Benro), 1989, Science nazie, science de mort. L'extermination des Juifs, des Tziganes et des malades mentaux de 1933 à 1945, trad. de l'allemand par Olivier Mannoni, Paris, Odile Jacob.

Olender (Maurice), 1989, Les Langues du paradis. Aryens et Sémites : un couple providentiel, préf. Jean-Pierre VeRnANT, Paris, Gallimard-Seuil.

PASPATI (Alexandros Georgios), 1870, Études sur les Tchinghianés ou Bohémiens de l'Empire ottoman, par Alexandre G. Paspati, Constantinople, Imprimerie Antoine Koroméla.

Pennell (Elizabeth Robins), 1893, To Gipsyland, Londres, T. Fisher Unwin.

PollaK (Michael), 2000, L'Expérience concentrationnaire. Essai sur le maintien de l'identité sociale, Paris, Métailié.

Pотт (August Friedrich), 1844-1845, Die Zigeuner in Europa und Asien. Ethnographischlinguistische Untersuchung, vornehmlich ihrer Herkunft und Sprache, nach gedruckten und ungedruckten Quellen, Halle, Haynemann, 2 vol.

PREDARI (Francesco), 1841, Origine e vicende dei Zingari, con documenti intorno le speciali loro proprieta fisiche e morali, la loro religione, i loro usi e costumi, le loro arti e le attuali loro condiziane politiche e civile in Asia, Africa ed Europa, con un saggio di gramatica e di vocabolario dell'arcano loro linguaggio di Francisco Predari, Milan, Tipografia di Paolo Lompato.

RENAn (Ernest), 1996, Qu'est-ce qu'une nation ?, et autres écrits politiques, introd. de Raoul GIRARDET, Paris, Imprimerie nationale (Acteur de l'Histoire).

Roche (Daniel), 1999, Leçon inaugurale au Collège de France faite le vendredi 19 novembre 1999, Paris, Collège de France.

RÜDIGER (Jacob Carl Christoph), 1782-1793, Neuerster Zuwachs der Teutschen fremden und allgemeinen Sprachkunde in eigenen Aufsätzen, Bücheranzeigen und Nachrichten, 5 Stücke, Leipzig-Halle, Rüdiger.

SAMPSON (John), 1907, « Gypsy language and origin », Journal of the Gypsy Lore Society (Liverpool), new ser., vol. I, p. 4-22.

SAMPSON (J.), 1911, « Jacob Bryant. Being an analysis of the Anglo-Romani vocabulary, with a discussion of the place and date of collection and an attempt to show that Bryant, not Rüdiger, was the earlest discoverer of the Indian origin of the Gypsies », Journal of the Gypsy Lore Society, new ser., vol. IV, 3, p. 162-194.

SAMPSON (J.), 1926, The Dialect of the Gypsies of Wales, being the older form of British Romani preserved in the speech of clan of Abram Wood, by John Sampson, Oxford, Clarendon Press.

SCHLEGEL (August Wilhelm von), 1823 et 1824, Indische Bibliothek, eine Zeitschrift von August Wilhelm von Schlegel, Bonn, bei Edward Weber, 2 vol.

SCHREIBER (Giorgio), 1935, «La stérilisation eugénique en Allemagne, une année d'application de la loi du 14 juillet 1935 pour l'enraiement de l'hérédité morbide », Revue anthropologique, année 45, p. 84-91.

SERgent (Bernard), 1995, Les Indo-Européens. Histoire, langues, mythes, Paris, Payot et Rivages (Bibliothèque scientifique Payot).

STEINER (George), 1969, Langage et silence, trad. de l'anglais par Lucienne LOTRINGER, Paris, Seuil. 
TURner (Ralph Lilley), 1927, The Position of Romani in Indo-Aryen, Londres, B. Quaritch.

VERMEIL (Edmond), 1948, Doctrinaires de la Révolution allemande, 1918-1938, Paris, Nouvelles éditions latines.

Willems (Wim), 1997, In search of the true Gypsy. From Enlightenment to the Final Solution, Londres, Frank Cass. 